\title{
Apropiación privada de la tierra y derechos políticos en la obra de John Locke
}

\author{
Joan SEVERO CHUMBITA* \\ Universidad de Buenos Aires \\ joanchumbita@gmail.com
}

\begin{abstract}
RESUMEN
A fin de considerar la influencia de la propiedad de bienes materiales sobre el ejercicio de los derechos políticos en la obra de John Locke, se analizará, en primer lugar, la distribución territorial y la medición en acres de los requerimientos para la participación política y el ejercicio de funciones públicas en The Fundamental Constitutions of Carolina; en segundo lugar, las consideraciones sobre la propiedad de la tierra como medio de producción y el trabajo salarial en el capítulo $\mathrm{V}$ de Two Treatises of Government, II; por último, se analizarán las restricciones patrimoniales para el acceso a derechos políticos así como la participación sugerida para los trabajadores en Some Considerations of the Lowering of Interest, and Raising the Value of Money.
\end{abstract}

PALABRAS CLAVE: Locke, apropiación, tierra, derechos políticos.

\begin{abstract}
In order to consider the influence of tangible property on the exercise of political rights in the work of John Locke, we'll analyze, first, the distribution and acreage measurement of the requirements for political participation and the exercise of public functions in The Fundamental Constitutions of Carolina; secondly, the considerations on land ownership, as a means of production, and the wage labor in Chapter $\mathrm{V}$ of Two Treatises of Government, II; finally, we'll analyze the patrimonial restrictions for the access to political rights, as well as the suggested participation for workers in Some Considerations of the Lowering of Interest, and Raising the $V$ alue of Money.
\end{abstract}

KEYWORDS: Locke, appropriation, land, political rights.

\section{Introducción}

La noción de propiedad privada en el capítulo quinto del segundo de los Two Treatises of Government definida como vida, libertad y bienes, alberga una ambigüedad manifiesta. Esta ambigüedad no representa un problema para comprender la génesis de esta teoría del derecho exclusivo a los bienes y, en especial, a la tierra. En efecto, los bienes son medios necesarios para la vida y su abundancia para la libertad. Lo mismo ocurre a la hora de comprender que la protección de estos derechos exclusivos sobre los bienes constituye el fin de la sociedad política. Sin embargo, al momento de establecer qué injerencia tiene la

\footnotetext{
* Este trabajo forma parte del proyecto de investigación UBACyT "La noción de política en el pensamiento de Giorgio Agamben, Roberto Esposito y Antonio Negri”, dirigido por el Dr. Marcelo Sergio Raffin, correspondiente al área de Sociología, Programación científica 2012-2015, de la Facultad de Ciencias Sociales de la UBA.
} 
propiedad privada y, más precisamente, la posesión de ciertos bienes materiales sobre la participación política, la ambigüedad del término requiere de una exégesis más exhaustiva.

En este sentido, no basta con recurrir al segundo tratado lockeano ${ }^{1}$, sino que es preciso considerar propuestas políticas concretas, en las que, como veremos, Locke se vuelve más explícito sobre la cuestión: son las contenidas en The Fundamental Constitutions of Carolina ${ }^{2}$ y en Some Considerations of the Lowering of Interest, and Raising the Value of Money ${ }^{3}$.

\section{Distribución territorial y cuantificación en acres de los derechos políticos en la Constitución de Carolina}

"El documento más importante de los papeles de Carolina es la Constitución Fundamental de Carolina, que establece una interesante mezcla de políticas liberales y jerarquías sociales restrictivas. Un diseño de nobleza fue inventado; a Locke se le concedió el segundo rango más alto, de «Landgrave» (probablemente un término acuñado por Locke), y los cuarenta y ocho mil acres que acompañan el título".

Wayne Glausser ${ }^{4}$

La Constitución de Carolina de 1669 cuenta con una versión escrita de puño y letra de Locke, en su calidad de secretario de los Señores Propietarios ${ }^{5}$. Este documento muestra la extraña combinación de una arquitectura económica colonial, una construcción social y política feudal, junto a la incorporación de ciertos elementos republicanos. La Constitución comienza por destacar la concesión de la provincia de Carolina por parte del rey Carlos II de Inglaterra a los Señores Propietarios ${ }^{6}$. Este territorio, según se estipula, será dividido en diferentes distritos, que habrían de conservar una misma proporción distributiva: doce mil acres de los Señores Propietarios, doce mil acres de la nobleza

\footnotetext{
${ }^{1}$ En J. LOCKE, J., Works of John Locke in Nine Volumes, t. IV, Londres, Rivington, 1824. Londres. De aquí en adelante citado como T. T., I para el primer tratado, T. T., II para el segundo. Todas las citas serán traducidas al castellano, conservando las referencias a la edición en su lengua original.

${ }^{2}$ J. LOCKE, Works of John Locke in Nine Volumes, t. IX, 175-199; J.LOCKE, Political writings [ed. D. Wootton], Indianapolis-Cambridge, Hackett Publishing Company, 2003, 210-232. De aquí en adelante citado como FCC.

${ }^{3} \mathrm{~J}$. LOCKE, Works of John Locke in Nine Volumes, t. IV, 1-116. De aquí en adelante: SC.

4 W. GLAUSSER, "Three Approaches to Locke and the Slave Trade". Journal of the History of Ideas. Vol. 51. No. 2 (Apr. - Jun.), 1990, 201.

5 "[...] hay un manuscrito de Locke, y muchas ediciones de sus obras que la incluyen" (W. GLAUSSER, "Three Approaches to Locke and the Slave Trade", 201). Como consigna correctamente Wootton: "Shaftesbury y Locke eran efectivos legisladores de Carolina" (D. WOOTTON, "John Locke and Richard Ashcraft's Revolutionary Politics", Political Studies, Vol. 40, March 1992, XL, 1992, 87). Estos elementos permiten establecer la autoría o, al menos la co-autoría de Locke sobre FCC.
} 
(barones, en su mayoría herederos de los Señores Propietarios o allegados de la máxima confianza) y doce mil acres para su colonización. Sin embargo, los doce mil acres de las colonias no serían exclusivamente cedidos a colonos libres, sino que con el fin de "preservar el equilibrio del gobierno", un quinto de esta extensión sería propiedad de los Señores Propietarios y otro quinto de la nobleza. Las restantes tres quintas partes de la colonia serían distribuidas entre los colonos libres, que habrían de pagar un tributo a los Señores Propietarios por detentar el dominio de la tierra y ejercer su actividad productiva en la provincia de Carolina?.

El carácter feudal de la organización social y política propuesta por la Constitución se evidencia en una multiplicidad de elementos configurados principalmente por los privilegios vitalicios y hereditarios de los Señores Propietarios y la nobleza, así como la constitución de un vasallaje igualmente hereditario asociado a las diferentes parcelas de tierra $^{8}$. En primer lugar, se destaca el carácter nobiliario y a perpetuidad de la cesión de Carlos II, así como la disposición de una estructura política basada en títulos de nobleza, coronados por la supremacía, de por vida, de ocho Señores Propietarios. De aquí, por ejemplo, que ningún Señor Propietario pueda ser removido de la corte que le toca presidir ${ }^{9}$. En el mismo sentido, se destaca que ningún hombre libre podrá ser sometidos a proceso alguno sino por sus pares ${ }^{10}$. En el caso de los miembros de la nobleza, deberán ser juzgados por la corte suprema de justicia y con un jurado conformado por sus pares ${ }^{11}$. La corte superior de justicia, por su parte, no considerará ningún proceso civil o penal que se halle en las cortes a cargo de alguno de los Señores Propietarios ${ }^{12}$. Por último, todas las

6 J. LOCKE, FCC en Political Writtings, 210-211, \$\$1-4.

7 J. LOCKE, FCC, $₫ 4$.

8 Capítulo aparte requiere la consideración del status jurídico de los esclavos según la Constitución, tanto en materia religiosa como en cuanto a su sometimiento absoluto al poder del amo en el orden "civil", J. LOCKE, FCC, \$107, \$110. Lo cierto es que los esclavos son considerados fundamentalmente como mano de obra para el trabajo en las colonias. Las referencias bibliográficas más destacadas sobre la cuestión, así como una consideración de los usos del término esclavitud en T. T., podrán encontrarse en J. CHUMBITA, "Un análisis de las nociones de abundancia y esclavitud para reinterpretar el carácter universal de la teoría de la apropiación de John Locke", Las torres de Lucca, $\mathrm{N}^{\circ}$ 2, enero-junio (2013b), 6983.

9 J. LOCKE, FCC en Political Writtings, 210-211; \$31.

$10 \mathrm{~J}$. LOCKE, FCC, $\$ 111$.

$11 \mathrm{~J}$. LOCKE, FCC, $\$ 27$.

$12 \mathrm{~J}$. LOCKE, FFC, $\$ 38$. 
instancias jurídico-políticas de apelación se hallan gobernadas, en última instancia, por la decisión última e inapelable de los Señores Propietarios ${ }^{13}$.

El reverso de la primacía social y política nobiliaria en la provincia de Carolina, es el vasallaje de por vida de todos los siervos, así como de sus hijos, "por todas las generaciones"14. Los siervos son asociados a un determinado dominio territorial y se estipula que requerirán de un permiso especial para trasladarse ${ }^{15}$. En el mismo sentido, la venta de terrenos destinados a la explotación colonial se realiza incluyendo a los siervos vinculados a tal dominio ${ }^{16}$.

A la primacía de los Señores Propietarios y su nobleza, así como al carácter igualmente hereditario del vasallaje, se añade el hecho de que las disposiciones de la Constitución son consideradas inalterables (sin nada semejante al derecho de resistencia que aparece en T. T., II ${ }^{17}$. Estos elementos dan cuenta, por tanto, del tipo de dominio ejercido por cesión real, de la casta de los ocho Señores Propietarios y sus herederos.

En cuanto al status de los colonos libres (freeholders), la Constitución estipula que no se permitirá a ningún habitante la compra o venta de tierras a los nativos de América sin la mediación de los Señores Propietarios, bajo pena de destierro a perpetuidad y confiscación completa de sus bienes ${ }^{18}$. En efecto, la situación de los colonos se halla muy lejos de la descrita en el capítulo $\mathrm{V}$ de T. T., II, donde se establece su independencia y derecho de apropiación a partir del trabajo, sin necesidad de mediar pacto político alguno ${ }^{19}$. Según la Constitución de Carolina sólo poseen derechos de propiedad en virtud de la concesión de los Señores Propietarios, a quienes deben pagar tributo a perpetuidad:

\$113. Todo aquel que posea cualquier propiedad $^{20}$ en Carolina, en virtud de su título o concesión, deberá como muy tarde a partir y después del año 1689, pagar anualmente a los Señores Propietarios, por cada acre de tierra, en medida inglesa, tanto de plata fina

13 J. LOCKE, FFC, $\$ 46, \$ 47$.

$14 \mathrm{~J}$. LOCKE, FFC, $\$ 23$.

$15 \mathrm{~J}$. LOCKE, FFC, $\$ 22$.

$16 \mathrm{~J}$. LOCKE, FFC, \$19.

17 J. LOCKE, FCC, $\$ 120$.

$18 \mathrm{~J}$. LOCKE, FCC, $\$ 112$.

19 J. LOCKE, T. T., II, \25-28, \50. Para un análisis pormenorizado de esta situación, véase J. CHUMBITA, "El desplazamiento en la teoría de la propiedad de John Locke: del criterio de necesidad a la teoría del valor para justificar la colonización inglesa en América", Mendoza, Cuyo. Anuario de filosofía Argentina y Americana, vol. 28 (2011), 93-120.

20 El término utilizado es "freehold". 
como posee en el presente un centavo inglés, o su valor correspondiente, en concepto de renta principal y reconocimiento a los Señores Propietarios, sus herederos y sucesores, por siempre ${ }^{21}$.

La centralidad económica de las colonias se evidencia no sólo en la mencionada distribución territorial por tercios entre Señores Propietarios, nobleza y tierras para la colonización, sino en la cláusula jurídica que prevé el progresivo cultivo agrario a partir del despliegue de la actividad de los colonos. La Constitución supone de este modo una expansión territorial que se basa principalmente en la incorporación de población de colonos y, en consecuencia, el incremento de la producción. La centralidad estratégica de las colonias se advierte a su vez en la prioridad otorgada a la extracción de la producción para su comercialización ${ }^{22}$. De aquí, por ejemplo, que la construcción de ciudades portuarias deba realizarse en los territorios de las colonias ${ }^{23}$.

Respecto a los elementos republicanos, hay que destacar la división de poderes, la institución de parlamentos conformados por los diferentes estamentos (Señores Propietarios, barones y colonos) y el carácter constitucional del ordenamiento jurídico colonial en su conjunto. Sin embargo, como se ha anticipado, los parlamentos en los distritos de los colonos, si bien cuentan con la potestad de proponer legislación, su aprobación se haya sujeta a la revisión y aceptación por parte del Gran Consejo (con mayoría de los Señores Propietarios, y poder de veto automático y definitivo) ${ }^{24}$. En el mismo sentido, todas las cortes judiciales menores dependen de la Corte Palatina, gobernada a su vez por el Señor Propietario Palatino (cargo que recaerá siempre en el Señor Propietario de mayor edad, ante la muerte de su predecesor) e integrada por los siete restantes Señores Propietarios ${ }^{25}$. Las otras siete cortes, a cargo de cada uno de los restantes siete Señores Propietarios, ocupadas de las diferentes áreas (relaciones exteriores, justicia, ejército, comercio, tesoro, administración, cultura) ${ }^{26}$, son igualmente inapelables y pueden fallar sobre cualquier causa que sea introducida en las cortes

21 J. LOCKE, FCC, \$113; véase también \$114.

$22 \mathrm{~J}$. LOCKE, FCC, $\$ 10$.

23 J. LOCKE, FCC, $\$ 94$.

$24 \mathrm{~J}$. LOCKE, FCC, \$50-51, \$53, \$75-77.

25 J. LOCKE, FCC, \$28, \$33, \$42, \$92, \$111.

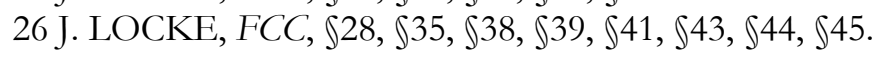


inferiores ${ }^{27}$. La Constitución de Carolina estipula, a su vez, que los miembros de los diferentes parlamentos, antes de tomar posesión de su banca y hallarse habilitados para votar, deben jurar suscribir a la Constitución ${ }^{28}$.

Sin embargo el aspecto que quizás mejor revela la estructura del ordenamiento jurídico propuesto, es el establecimiento de la cantidad de acres como condición del ejercicio de derechos políticos. En efecto, este aspecto da cuenta de los tres elementos mencionados: el carácter estamental del ordenamiento social y político, el diseño económico colonial así como el carácter formal de los elementos republicanos:

Ningún hombre deberá estar registrado en una colonia sin poseer más de cincuenta acres en propiedad ${ }^{29}$ dentro de dicha colonia."30 "Ningún hombre deberá ser registrado en ningún distrito con menos de trescientos acres en propiedad dentro de dicho distrito ${ }^{31}$.

La Constitución estipula a su vez que aquel que se postule como agente o suboficial de policía de una colonia debe contar con cien acres de tierra ${ }^{32}$. La postulación a juez y administrador en la corte del distrito demanda contar con trescientos acres. Para apelar el fallo de esta corte y ser recibido en la corte del condado (ordenamiento jurídico superior al del distrito local), se estipula el pago de cincuenta libras ${ }^{33}$. Ser jurado de la corte de distrito requiere poseer cincuenta acres de tierra en propiedad libre; ser jurado de la corte del condado la posesión de al menos trescientos acres; ser jurado de la corte del Señor Propietario exige poseer quinientos acres ${ }^{34}$. Postularse como representante del parlamento del distrito a las bancas destinadas a los $\operatorname{colonos}^{35}$ (la mayoría se haya ya garantizada para los Señores Propietarios y representantes de la nobleza), requiere contar con al menos quinientos acres. Votar en la elección de estos representantes de los colonos, demanda la posesión libre de al menos cincuenta acres ${ }^{36}$.

27 J. LOCKE, FCC, \$46-47.

$28 \mathrm{~J}$. LOCKE, FCC, $\$ 74$.

29 El término utilizado es "freehold".

$30 \mathrm{~J}$. LOCKE, FCC, $\$ 85$.

$31 \mathrm{~J}$. LOCKE, FCC, $\$ 82$.

$32 \mathrm{~J}$. LOCKE, FCC, $\$ 91$.

$33 \mathrm{~J}$. LOCKE, FCC, $\$ 67$.

$34 \mathrm{~J}$. LOCKE, FCC, $\$ 68$.

35 El término utilizado es "freeholders".

36 J. LOCKE, FCC, \$71-72; R. BECKER, “The ideological commitment of Locke: Freemen and servants in the Two Treatises of government". History of Political Thought, Vol. XIII, N. 4, Winter (1992), 654-655. 
De este modo, la Constitución de Carolina refleja la aspiración de realizar una explotación económica expansiva a través del cultivo progresivo de las tierras de la provincia. En este sentido, se incorporan formas precarias de derechos políticos para los colonos, que más bien parecen de carácter formal en la medida en que dependen siempre en última instancia de la decisión superior, con potestad revocatoria, de los Señores Propietarios y la nobleza. Al mismo tiempo, estos derechos de representación política minoritaria se establecen bajo la condición de validar una distribución de la tierra estructuralmente desigual e inmodificable.

Por todo lo dicho, podemos extraer como conclusión que existe en la Constitución de Carolina una relación directa entre la posesión de determinada extensión territorial y el acceso a derechos políticos.

\title{
La tierra como medio de producción y el trabajo a cambio de un salario
}

\begin{abstract}
"Independientemente de cualquier otra cosa que pudiera ser, el capítulo V es un argumento significativo para justificar el cercamiento en Inglaterra. Más allá de esto, sin embargo, es un razonamiento ingenioso a favor del colonialismo, defendiendo implícitamente el establecimiento de colonias y plantaciones en América y en otras partes, y el cercamiento y la mejora de la tierra sin cultivar en esos territorios vírgenes".

Neal Wood ${ }^{37}$
\end{abstract}

Tanto si consideramos el capítulo V de T. T., II, como FCC o determinados pasajes de SC, es claro que para Locke el trabajo de la tierra ocupa un lugar privilegiado en su concepción económico-política. El trabajo de la tierra es distintivo de los industriosos y racionales y sirve de basa a toda la teoría de la propiedad. Para entender la centralidad de la tierra en la teoría de la propiedad y de ésta como criterio restrictivo de los derechos políticos, es preciso entender, en primer lugar, que la tierra es para Locke un medio de producción, $y$, en este sentido, mediada por el trabajo, se constituye en fuente de la riqueza. De aquí surge su preeminencia política: "La cuestión principal de la propiedad no es hoy la de los frutos de la tierra, ni la de las bestias que subsisten en ella, sino la de la tierra en sí misma, dado que contiene y da sustento a todo el resto" 38 .

37 N. WOOD, John Locke and Agrarian Capitalism, Berkeley, University of California Press, 1983, 66.

38 J. LOCKE, T. T. II, §32. Véase J. TULLY, A discourse on Property: John Locke and Adversaries, Cambridge: Cambridge University Press, 1980, 119. 
La propiedad de la tierra es la cuestión fundamental de la propiedad. Esta afirmación no puede dejar de subrayarse. La tierra contiene por un lado los frutos necesarios para la vida, y es el medio a través del cual el trabajo produce nuevos bienes y se hace posible, según Locke, el progreso económico y social ${ }^{39}$. Sin embargo, aún cuando la tierra es medio de producción, carece casi por completo de valor sin la mediación del trabajo humano ${ }^{40}$.

Si bien la tierra es la fuente de los frutos, una tierra no trabajada o cuyo producto no pueda ser comercializado por su ubicación remota, carece de valor. Aquí la valorización de la tierra atiende tanto a su valor de uso como al valor de cambio de lo que produce. En efecto, respecto al valor de uso, se consideran los bienes que una tierra es capaz de producir a través de la mediación del trabajo. Pero este valor de uso, esta productividad, siempre se halla sujeta al valor de cambio del producto, determinado por la capacidad de extraer y comercializar la producción obtenida. Siguiendo el ejemplo lockeano, una tierra en el interior de América, aún cuando albergue una alta capacidad productiva, carece de valor si su fruto no puede ser transportado a un mercado de consumidores ${ }^{41}$.

Por lo tanto dos parcelas ubicadas de modo tal que su producción resultara igualmente transportable a un mercado de consumidores, no tendrán el mismo valor si una hubiese recibido los beneficios de treinta años de labranza y la otra se hallara desprovista de esta mejora: "Pues, verdaderamente, es el trabajo lo que les confiere a todas las cosas su valor diferencial" ${ }^{42}$. El valor de la tierra surge entonces de su valor de uso, siempre y cuando sea posible realizar el ciclo de la producción en condiciones similares. De este modo queda claro que la tierra es considerada como un medio de producción, puesto que su valor surge no sólo de sus cualidades intrínsecas ni del trabajo acumulado en ella sino de su productividad real, de su condición efectiva como medio para realizar el ciclo productivo.

39 J. LOCKE, T. T. II, \$34.

$40 \mathrm{~J}$. LOCKE, T. T. II, \$36-37, \$44.

$41 \mathrm{~J}$. LOCKE, T. T. II, $\$ 48$.

42 J. LOCKE, T. T. II, \40; véase también $\$ 41-43$; véase K. OLIVECRONA, "Locke's Theory of Appropiation”, The Philosophical Quarterly, Vol. 24., Nro. 96, Julio, 1974, 220. 
Es posible que la tierra sea más estéril, y por lo tanto, el producto es menor y, en consecuencia el dinero que se recibe por ese producto es también menor. Porque es evidente que aquel cuya tierra solía producir 100 bushels de trigo communibus annis, si por su cultivo prolongado o por su mala administración produce ahora 50 bushels, la renta se verá reducida a la mitad ${ }^{43}$.

El hecho de que la tierra sea considerada como medio de producción se advierte, a su vez, cuando Locke señala que la apropiación de tierras a través del trabajo no reduce sino que incrementa el acervo común de la humanidad ${ }^{44}$. Esto se debe a que, como bien explica Waldron, por medio del cercamiento y la agricultura se utiliza menor cantidad de tierra para producir una determinada satisfacción de necesidades humanas, de la que se requeriría por medio del uso sin cercamiento, mediante la caza o la recolección ${ }^{45}$. De aquí que mientras persista esta proporción, no importa de cuánto se apropie alguien mediante el trabajo pues, lejos de reducir el acceso a los bienes, el uso de la tierra como medio de producción beneficiará a la humanidad en su conjunto por el incremento de bienes disponibles ${ }^{46}$.

Consideradas estas razones, junto a la centralidad otorgada a la tierra en el capítulo $\mathrm{V}$ de T. T., II, no es de extrañar que Wood haya definido la teoría lockeana de la propiedad en términos de capitalismo agrario. De este modo, Wood recupera críticamente la interpretación de Macpherson ${ }^{47}$. En efecto, según Wood en la obra de

43 J. LOCKE, Escritos monetarios [tr. María Olaechea], Madrid, Pirámide, 116; véase también 89.

44 J. LOCKE, T. T. II, \$37.

45 Acerca de la promoción del cercamiento en la obra de Locke, véase J. MARSHALL, John Locke. Resistance, religion and responsibility, Cambridge, Cambridge University Press, 1994, 281-282; N. WOOD, John Locke and Agrarian Capitalism, 66, 95.

46 J. WALDRON, "Enough and as Good Left for Others". Philosophical Quarterly. 29 (1979), 323; J. TULLY, $A$ discourse on Property, 123. Para una crítica a esta reivindicación, véase C. B. MACPHERSON, La teoría política del individualismo posesivo. De Hobbes a Locke [tr. J.-R. Capella], Barcelona, Fontanella, 1970, 183184.

$47 \mathrm{La}$ interpretación de Macpherson tiene el mérito de introducir a la exégesis de la teoría de la apropiación lockeana (en sintonía con las afirmaciones de Strauss [1992]), la presencia de sujetos con intereses enfrentados. Sin embargo, adolece, como bien ha establecido Wood, de una proyección de actores del siglo XIX (burguesía y proletariado industrial) al siglo XVII. En este sentido, el antagonismo en el capítulo $\mathrm{V}$ de $T$. T., II, surge más bien entre una burguesía enfrentada tanto a nativos en las colonias, esclavos, pobres y una monarquía sin regulación parlamentaria en Inglaterra (B. ARNEIL, "John Locke, Natural law and colonialism", History of Political Thought, Vol. XIII, N. 4, Winter (1992); D. ARMITAGE, "John Locke, Carolina, and the Two Treatises of Government", Political Theory, 32, (2004), 602; R. BECKER, "The ideological commitment of Locke", (1992), 654-655; J. CHUMBITA, "El desplazamiento en la teoría de la propiedad de John Locke"; J. CHUMBITA, "La caridad como administración de la pobreza", Identidades, Revista del Instituto de Estudios Sociales y Políticos de la Patagonia, No 4, junio (2013a), 1-21; 
Locke encontramos los componentes fundamentales de la definición marxista de capital: la división de clases entre quienes poseen los medios de producción y quienes por carecer de ellos se ven obligados a vender su fuerza de trabajo. La fuerza de trabajo es de este modo comprada como mercancía para obtener un plusvalor o, dicho en términos lockeanos, una ganancia o maximización de beneficios ${ }^{48}$. Sin embargo, diferenciándose de Macpherson, como Wood destaca acertadamente, la noción de capital que podemos encontrar en Locke no debe identificarse con el capitalismo industrial al que se refería Marx a partir del estudio de las condiciones industriales y urbanas del siglo XIX ${ }^{49}$.

Resulta adecuado, por tanto, aplicar a los desarrollos lockeanos sobre la tierra una descripción en términos de medio de producción y, en consecuencia, de capital. Sin embargo, la interpretación de la teoría de la propiedad al interior de un modo de producción capitalista exige, además, dar cuenta de una consideración del trabajo como mercancía. En este sentido, uno de los elementos menos evidentes en el capítulo $\mathrm{V}$ de $T$. T., II, es la enajenabilidad del fruto del trabajo. Sin embargo, el siguiente pasaje permite inferir que Locke reconoce efectivamente esta posibilidad:

[...] la hierba que mi caballo ha mordido, el césped que mi sirviente ha cortado y el mineral que he extraído de la tierra, en cualquier lugar en el que tenga derecho a ellos en común con otros, se convierten en mi propiedad sin la concesión o el consentimiento de nadie $^{50}$.

En la medida en que pertenece al dueño del caballo la hierba con que éste se alimenta, también pertenece el producto del trabajo del siervo a quien compra su tiempo con un

para la importancia de América en la teoría de la propiedad, véase especialmente R. TUCK, The Rights of War and Peace: Political Thought and the International Order from Grotius to Kant, Oxford, Oxford University Press, 2009, 166.

48 N. WOOD, John Locke and Agrarian Capitalism, 19.

49 Wood expone observaciones más que pertinentes respecto al anacronismo propio de la interpretación de Marcpherson: "Él parte de la premisa fundamental de que la Inglaterra del siglo XVII era una sociedad de mercado, sin embargo, en ninguna parte demuestra la validez histórica de esta afirmación, una equivocación decisiva, considerando las dudas de muchos comentaristas sobre la cuestión" (N. WOOD, John Locke and Agrarian Capitalism, 7). Tully había dado razones similares para rechazar la interpretación de Macpherson. Sin embargo, su interpretación es completamente diferente: "en Two Treatises, Locke ofrece una justificación, no de la propiedad privada, sino que, más bien, de la comuna inglesa" (J. TULLY, $A$ discourse on Property, 130, véase también 124-125).

$50 \mathrm{~J}$. LOCKE, T. T. II, $\$ 28$. 
jornal ${ }^{51}$. Podría cuestionarse la interpretación de Wood por suponer el contrato salarial en esta alusión marginal a la apropiación del trabajo del siervo. Sin embargo, la enajenación del producto del trabajo a cambio de un salario es contemplada en otros pasajes de T. T., II que explícitamente refieren a la relación servil: "un hombre libre se hace a sí mismo siervo de otro vendiéndole, por un cierto tiempo, el servicio que se compromete a brindar en contraprestación por los jornales que ha de recibir" 52 . De aquí se sigue también el carácter libre de este contrato laboral, lo cual permite interpretarlo como relación salarial capitalista.

Tully rechaza esta interpretación, sosteniendo que es "lógicamente imposible que un agente aliene su trabajo"53. La afirmación de Tully elude considerar la referencia explícita y concreta al producto del trabajo del siervo que es sustraído a cambio de un jornal, para problematizar la coherencia interna del concepto de alienación. Esta operación resulta aún más sorprendente si observamos que Tully analiza el parágrafo \$85 que acabamos de citar. En efecto, en este parágrafo aparece no sólo la referencia mencionada al trabajo salarial y servil, sino que es considerada también la figura de esclavitud legítima, como el propio Tully advierte. Ahora bien, si resulta absurdo negar la posibilidad de la alienación del trabajo en la relación salarial, tanto más lo es en la figura de esclavitud legítima, la cual manifiestamente establece una relación de trabajo forzoso, cuyo fruto es enajenado ${ }^{54}$. En el mismo sentido, podemos consignar el trabajo forzoso en las casas de trabajo propuesto en la ley de pobres ${ }^{55}$ y el trabajo del siervo y el esclavo en la Constitución de Carolina ${ }^{56}$. De este modo, más allá de la discusión sobre si es "lógica", o más bien coherente, en sí misma, la idea de alienación del trabajo, es claro que, al interior de la teoría de la propiedad lockeana descrita en T. T., II, el fruto del trabajo es

51 N. WOOD, John Locke and Agrarian Capitalism, 88.

52 J. LOCKE, T. T. II, $\$ 85$.

$53 \mathrm{~J}$. TULLY, $A$ discourse on Property, 138. Tully, no sólo sostiene que no hay trabajo enajenado en la relación entre el amo y el sirviente referida en J. LOCKE, T. T., II, \$28 y $\$ 85$ (J. TULLY, $A$ discourse on Property..., 135) sino que niega la posibilidad del capitalismo al interior de la teoría de la propiedad lockeana (J. TULLY, $A$ discourse on Property, 137). Incluso sugiere que Locke no intenta justificar la apropiación privada (como surge explícitamente de J. LOCKE, T. T., II, $\$ 25$ y \$50, como objetivo del capítulo V), sino una "teoría de una propiedad natural sobre los productos del trabajo propio y usada para legitimar rebeliones contra el sistema imperante de propiedad privada" (J. TULLY, A discourse on Property..., 124).

54 J. LOCKE, T. T., II, \$85, \$23, \$24. Véase J. CHUMBITA, “Un análisis de las nociones de abundancia y esclavitud...", 69-83.

55 Para una consideración detallada de Draft, véase J. CHUMBITA, "La caridad como administración de la pobreza", 12-18.

$56 \mathrm{~J}$. LOCKE, FCC, $\$ 22$. 
perfectamente extraíble al siervo en virtud de la compra de su fuerza de trabajo a cambio de un jornal.

Habiendo establecido que en T. T., II, la tierra es considera como medio de producción y el trabajo como mercancía, se abordará finalmente la cuestión de los requisitos patrimoniales para el ejercicio de los derechos políticos normales así como el lugar reservado a los trabajadores en $S C$.

\section{Requisitos patrimoniales y participación política de los trabajadores}

“[...] disputas sobre los títulos, los límites, la usurpación, el cercamiento, la herencia, etc. Estas, por supuesto, eran cuestiones que interesaban profundamente y afectaban directamente al magnate Whig a quien Locke servía. Por lo demás, ubicando el soberano supremo o el poder de hacer las leyes en el Parlamento, sujeto únicamente a la aprobación de la comunidad de propietarios, Locke aseguró que sus pares y los terratenientes tuvieran firmemente el control".

Neal Wood ${ }^{57}$

En este apartado se considerará la relación entre patrimonio y ejercicio de derechos políticos en T. T., II, así como el lugar que Locke otorga a la participación de los trabajadores. En este sentido, resulta fundamental interpretar la afirmación lockeana según la cual el fin de la sociedad política es la protección de la propiedad privada, así como establecer qué requisitos se exigen, por ejemplo, para detentar el derecho al voto y para postularse como representante del parlamento en la cámara de los comunes.

Según Tully, acorde con su interpretación de que la teoría de la apropiación lockeana no tiene como modelo la propiedad privada sino comunal ${ }^{58}$ y que la sociedad civil cuenta con la prerrogativa de redistribuir la apropiación privada ${ }^{59}$, Locke propondría en T. T., II el sufragio universal.

57 N. WOOD, John Locke and Agrarian Capitalism, 95.

58 J. TULLY, $A$ discourse on Property..., 130.

59 " [...] La explicación teórica de cómo la propiedad debe ser convencionalmente distribuida de acuerdo con la ley natural y los derechos naturales" (J. TULLY, A discourse on Property..., 130). Como bien advierte Waldron, la operación fundamental de Tully es manipular la idea de "regulación" para distorsionar lo que es la obviedad más explícita del capítulo V de T. T., II: la intención de establecer una propiedad privada desigual sin necesidad de consenso social ni político (J. LOCKE, T. T., II, \$25; J. WALDRON, "Locke, Tully and the Regulation of Property", Political Studies, XXXII, 1984, 98). Tully retoma los fragmentos en los que Locke sostiene que el pasaje a la sociedad civil implica que todas las posesiones del hombre pasan a estar reguladas por la comunidad, para inferir que esto implica una "distribución de la propiedad [ya que esta] es ahora convencional" (J. TULLY, $A$ discourse on Property..., 165). Sin embargo, esta interpretación carece de base textual. Locke reitera a lo largo de $T$. T., II que el fin de la sociedad civil es la protección de 
En demostración de que todo hombre tiene la propiedad de su vida, libertad, persona, acción y algunas posesiones, Locke extiende el voto a todos los varones adultos. Él no explicita este criterio en Two Treatises, sino que simplemente lo asume como base de su análisis de los diversos tipos de representación: «toda vez que el pueblo elija a sus representantes conforme a medidas innegablemente justas e iguales que se ajusten a la forma original de gobierno, no podrá dudarse de que se trata de la voluntad y de un acto de la sociedad» (T. T., II, \$158). Las medidas iguales basadas en la constitución original no pueden ser sino la igualdad natural de todos los hombres $(T \text {. T., II, } \ 5)^{60}$.

En primer lugar hay que destacar junto a Tully que Locke en ningún momento explicita cuál es el criterio de acceso al derecho tanto de postularse como representante como de elegir representantes de la cámara de los comunes. Por lo tanto, la afirmación de que abogaría por un sistema de representación universal carece de mayor sustento que el esgrimido en este pasaje: sostener que Locke lo da por supuesto. Lo cierto es que en $T$. T., II no hay referencia alguna al sistema electoral para la elección de parlamentarios de la Casa de los Comunes ${ }^{61}$. Tully se propone entonces fundar su interpretación construyendo un antagonismo entre un Locke democrático y un Filmer a favor del sistema de voto restringido por la posesión de propiedad, sin esgrimir otras razones de por qué Locke sostendría un sistema de representación universal ${ }^{62}$.

En este sentido, se ha subrayado la significación de este silencio sobre la extensión del sufragio, puesto que la cuestión era explícitamente planteada por los Levellers ${ }^{63}$. El argumento de Marshall es que si Locke hubiese abogado por un tipo de participación amplia, a diferencia de la legislación vigente, no habría dejado de explicitarla. El hecho de

la propiedad privada (vida, libertad y bienes), legítima con anterioridad a la fundación del pacto (J. LOCKE, T. T., II, \$85, \$138-140, \$171, \$222, \$240).

$60 \mathrm{~J}$. TULLY, A discourse on Property..., 173.

61 D. WOOTTON, "John Locke and Richard Ashcraft's Revolutionary Politics", 87.

$62 \mathrm{~J}$. TULLY, A discourse on Property, 173-174.

63 "Este silencio es extremadamente significativo: no había una teoría de la necesidad de la participación como expresión de la propiedad y personalidad de cada individuo, como se había implicado en los escritos del Leveller Overton a mediados de siglo, o de la necesidad de la democracia en favor de la que otros Levellers en ciertos momentos habían argumentado más explícitamente" (MARSHALL, John Locke. Resistance, religion and responsibility, 271; véase también D. WOOTTON, "John Locke and Richard Ashcraft's Revolutionary Politics", 97, M. SELIGER, "Locke's Theory of Revolutionary Action", The Western Political Quarterly, 16 (3) (sept., 1963), 548, 553; J. H. FRANKLIN, John Locke and the theory of sovereignty. Mixed Monarchy and the Right of Resistance in the Political Thought of the English Revolution, London, Cambridge University Press, 58; M. FOUCAULT, Defender la sociedad [tr. Horacio Pons], Buenos Aires, FCE, 2001, 6364; 105-106). 
que no lo hiciera, indicaría que se inclinaba por el sistema imperante o, cuanto menos, deja en claro que no lo cuestionaba.

Wood reconstruye en este sentido las restricciones imperantes en el sistema electoral: "en la época de la Revolución Inglesa el voto definitivamente se había ampliado." ${ }^{44}$ Luego de 1644, del total de la población masculina adulta, "eran elegibles para votar entre el 27 y el 40 por ciento." 65 Sin embargo, después de la revolución "la posición de la aristocracia terrateniente se había endurecido contra la ampliación del voto, que poco a poco se hizo más restringido"66.

El propio Tully reconoce que "el criterio convencional para el derecho de sufragio en el siglo XVII era la posesión de la propiedad"67. En el año 1679, durante la Crisis de exclusión, probablemente contemporánea al proceso de redacción de T. T., II (Ashcraft, 1980: 437; Marshall, 1994: 238), legisladores del partido whig, del que Shaftesbury era un miembro eminente, intentaron infructuosamente reformar el sistema electoral, para limitar el poder del rey. Se proponía reducir los cuarenta chelines en propiedad "limitando el voto del condado [county] a personas con patrimonios de al menos un valor de $f_{200 " 68}$. En el caso de los distritos [borough], el voto se extendería "a todos los que pagaran impuestos" pero "no necesariamente según un sistema más democrático" que en casos ya existentes, como las elecciones urbanas ${ }^{69}$. De este modo, aún cuando la propuesta de Shaftesbury apuntara a romper con las barreras arbitrarias de elección en los municipios y "la adopción de una votación uniforme" así como la "reforma del requisito de dominio absoluto cuarenta chelines para corresponder a la corriente equivalente en valor", sin embargo, lejos de "hacer que la representación en la base fuera lo más amplia posible", como interpreta Tully, apuntaba a "extender el sufragio horizontalmente y no verticalmente, es decir, incluir más personas en las categorías ya representadas" pero "no extenderlo sensiblemente hacia abajo para incluir categorías sin representación"70. De

64 N. WOOD, John Locke and Agrarian Capitalism, 84.

65 N. WOOD, John Locke and Agrarian Capitalism, 84.

66 N. WOOD, John Locke and Agrarian Capitalism, 95.

$67 \mathrm{~J}$. TULLY, A discourse on Property, 173.

68 N. WOOD, John Locke and Agrarian Capitalism, 84-85.

69 N. WOOD, John Locke and Agrarian Capitalism, 84-85. "En Londres, los 4.000 hombres libres con derecho a voto eran poco menos que el total de hombres libres. En York, el electorado era de alrededor de un 75 por ciento de la población masculina adulta, y en las tres otras ciudades cerca de 50 por ciento" (N. WOOD, John Locke and Agrarian Capitalism, 84-85; véase también D. WOOTTON, "John Locke and Richard Ashcraft's Revolutionary Politics", 94).

70 N. WOOD, John Locke and Agrarian Capitalism, 85. 
hecho, establecía estándares muy altos de riqueza y calificación para los postulantes a diputados e incluso en ciertos casos se elevaba "la calificación de dominio absoluto de cuarenta chelines para los votantes del condado, que habría significado una reducción del número previo de electores"71. A partir de estos elementos, Wood concluye, en virtud de la vinculación directa entre Shaftesbury y Locke, que no hay "nada de lo dicho por Locke que indicara que iría más allá de todo esto" 72 .

Más allá de la validez de estos argumentos sobre los silencios de T. T., II, hay una serie de elementos que nos permiten arrojar cierta claridad. En primer lugar, tener en consideración el criterio indudablemente patrimonial del sistema de representación parlamentario de la Constitución de Carolina. En segundo lugar, sin olvidar que en T. T., II, la cuestión del sistema de sufragio no es tratada de modo directo, resulta posible, sin embargo, interpretar determinadas referencias sobre la noción de propiedad privada y vincularlas con el ejercicio de los derechos políticos. Por último, recuperar un pasaje de $S C$ que resulta particularmente significativo.

Como hemos destacado, Locke utiliza en T. T., II el término propiedad privada con una ambigüedad explícita: propiedad es vida, libertad y bienes. Un ejemplo del uso amplio del concepto de propiedad privada, que incluye estas tres acepciones, se halla en los pasajes en que Locke legitima la resistencia del cuerpo social en su conjunto, considerando como fin de la sociedad civil, no sólo la protección de los bienes sino de la libertad o la vida de los súbditos ${ }^{73}$. Sin embargo, como hemos podido ver a lo largo de esta segunda parte, este no es el uso dominante ni tampoco es una afirmación que niegue en absoluto los requisitos patrimoniales para las elecciones parlamentarias de la Casa de los Comunes.

“[...] los hombres en sociedad, teniendo propiedad, tienen ese derecho a los bienes, los cuales, por la ley de la comunidad son suyos, y ningún cuerpo tiene el derecho de tomar

71 N. WOOD, John Locke and Agrarian Capitalism, 85. Para la denunciada este tipo de restricciones, véase C. SCHMITT, Teoría de la Constitución [tr. F. Ayala], Madrid, Alianza, 1992, 18, 33-36, 38, 40, 95-96, 137; C. SCHMITT, Sobre el parlamentarismo [tr. Thies Nelsson y Rosa Grueso], Buenos Aires, Tecnos, 1990, 13, 1618, 22; C. SCHMITT, Legitimidad y legalidad [tr. José Díaz García], Buenos Aires, Struhat \& Cía, 1994, 3940.

72 N. WOOD, John Locke and Agrarian Capitalism, 85. En favor de la interpretación del sufragio restringido, D. WOOTTON, "John Locke and Richard Ashcraft's Revolutionary Politics", 94-97; M. SELIGER, "Locke's Theory of Revolutionary Action", 548.

73 J. LOCKE, T. T., II, \$209, 221; véase también \$85, \$123. 
su patrimonio o parte alguna de él, sin su propio consentimiento [...]. Es por esto un error pensar que el poder supremo o legislativo de algún Estado puede hacer su voluntad y disponer de las pertenencias del súbdito arbitrariamente o tomar parte alguna de ellas a su antojo" ${ }^{, 74}$.

Este pasaje permite apreciar que, contrariamente a ciertas interpretaciones eminentes ${ }^{75}$, cuando Locke afirma que el fin de la sociedad civil es la protección de la propiedad privada, se refiere eminentemente a la protección del dominio sobre ciertos bienes ${ }^{76}$. La protección de la que se habla aquí es de "bienes" y, más precisamente, del "patrimonio"77. Claramente el problema de este pasaje es la confiscación de los bienes por parte del Estado. El ejemplo subsiguiente, del jefe militar que pude ordenar una maniobra en que esté en riesgo la vida del soldado pero no puede apropiarse ni un céntimo de su salario, no deja lugar a dudas acerca de que el problema radica en la confiscación de la propiedad en relación a los bienes ${ }^{78}$. Cuando Locke afirma que un heredero se halla vinculado a las leyes del Estado en el que su propiedad se halla asentada, y por lo tanto da consentimiento tácito a éstas, no está hablando de su libertad o su vida, o la vestimenta del mendigo o alguna que otra herramienta del sirviente asalariado, sino de los bienes adquiridos por medio de herencia ${ }^{79}$. De modo que, aún cuando estas referencias no permiten inferir directamente un criterio patrimonial para la pertenencia a la sociedad civil, ni, claro está, dejar de lado las otras dos acepciones de propiedad (como son la vida y la libertad) resulta claro que la preocupación central al afirmar que el fin de la sociedad civil es la protección de estos bienes patrimoniales.

En el mismo sentido, pero mucho más significativo y directo a los fines de mostrar la restricción de los derechos políticos, resulta el siguiente pasaje de SC, publicado

74 J. LOCKE, T. T., II, \138; véase \$131, \$139-140, \$142, \$171 y especialmente \$222; \$240.

75 Laslett (en J. LOCKE, Two Treatises of Government [tr. Peter Laslett], Cambridge, Cambridge University Press, 3-126), W. KENDALL, John Locke and the doctrine of the majority-rule, Illinois, University of Illinois Press, 1965; J. TULLY, A discourse on Property; R. ASHCRAFT, "Revolutionary Politics and Locke's Two Treatises of Government: Radicalism an Lockean Political Theory", Political Theory, vol. 8, $\mathrm{N}^{\circ}$ 4, (nov., 1980), 429-486.

$76 \mathrm{Al}$ respecto M. SELIGER, "Locke's Theory of Revolutionary Action"; N. WOOD, Jobn Locke and Agrarian Capitalism; D. WOOTTON, "John Locke and Richard Ashcraft's Revolutionary Politics", Arneil (1992); D. ARMITAGE, "John Locke, Carolina, and the Two Treatises of Government", han hecho aportes más que significativos. Véase J. CHUMBITA, "El desplazamiento en la teoría de la propiedad de John Locke".

77 J. LOCKE, T. T., II, §85; \131, \$142.

78 J. LOCKE, T. T., 139; véase también \$176, \182, \$184, \$193. 
contemporáneamente a T. T., II, en el que explícitamente Locke se refiere al tipo de discusión que tiene lugar en el ámbito parlamentario, así como las consecuencias de su mal ejercicio.

Esta tensión y competencia se producen habitualmente entre el propietario de tierras y el comerciante. Como la cuota de dinero ${ }^{80}$ que comparte el trabajador rara vez supera la mera subsistencia, nunca brinda tiempo ni oportunidad a ese grupo de hombres para dirigir sus pensamientos más allá de eso o para pelear junto con los que son más ricos que ellos por la suya [su parte ${ }^{81}$ ] (como un interés común), salvo cuando un gran problema común que los une en una agitación universal les hace dejar de lado el respeto y les da coraje como para ayudarse en sus necesidades con una fuerza poderosa, entonces, a veces caen sobre los ricos y arrasan con todo como un diluvio. Pero esto raramente sucede salvo cuando existe una mala o engañosa administración por parte de gobiernos negligentes ${ }^{82}$.

El pasaje parte del supuesto de que la discusión parlamentaria compete a quienes deben discutir la promoción de la actividad productiva de sus diferentes sectores. Estos hombres deben disponer del ocio para la formación en este tipo de discusiones, el cual requiere de “dinero". De aquí que los trabajadores no sean considerados para el ejercicio parlamentario. Éstos no se hallan formados como para participar de las discusiones públicas sobre las cuestiones de Estado que se dirimen en el parlamento ${ }^{83}$. Su participación se restringe, de este modo, a los momentos de crisis en que surge la resistencia civil, precisamente cuando la mala administración llega al punto de amenazar su derecho a los medios de subsistencia ${ }^{84}$.

79 J. LOCKE, T. T., II, \$121-122.

80 En realidad, "the share" (J. LOCKE, SC, 1824, t. IV, 71).

81 Remita a "the share", la parte o "cuota" del trabajador: connotando que el trabajador no tiene tiempo ni oportunidad de pelear por su parte correspondiente en cuanto trabajador (J. LOCKE, SC, 1824, t. IV, 71).

$82 \mathrm{~J}$. LOCKE, Escritos monetarios, 118.

83 Véase en este sentido la recopilación de referencias de Locke a los trabajadores en N. WOOD, John Locke and Agrarian Capitalism, 75. Para un análisis de la consideración que reciben los mendicantes en la propuesta de Locke de ley de pobres, véase J. CHUMBITA, "La caridad como administración de la pobreza". 12-17.

84 Seliger realiza esta interpretación en "Locke's Theory of Revolutionary Action", 551. Acerca de la participación de los trabajadores en la resistencia véase J. CHUMBITA, "La resistencia social como configuración del pueblo según John Locke”, Revista SAAP, Vol. 8, N 1, mayo 2014 (en prensa). Sobre el derecho universal a la vida, véase J. CHUMBITA, "La caridad como administración de la pobreza", 1-21. 


\section{Conclusiones}

Como hemos podido ver, la influencia de la propiedad privada, en especial como medio de producción, tiene una importante injerencia en la teoría política lockeana en general y en la consideración de los asuntos públicos, que se traduce a su vez en la asignación de derechos políticos. En este sentido, al considerar FCC, hemos dado cuenta del diseño territorial con un criterio económico evidente, así como la medición en acres de los requerimientos para la participación política y el ejercicio de funciones públicas. Hemos visto a su vez, en segundo lugar, la consideración en el capítulo V de T. T., II, de la tierra como la cuestión principal de la propiedad privada, en tanto es medio de producción, así como el reconocimiento del trabajo asalariado. Por último, a partir de SC y T. T., II, se ha intentado clarificar qué quiere decir Locke al sostener que el fin de la sociedad política es la protección de la propiedad privada, subrayando la restricción de la participación política de los trabajadores al momento de la resistencia.

De este modo, es posible concluir que a pesar de la consideración del trabajo como fuente de la riqueza ${ }^{85}$, se observa a lo largo de la obra lockeana una persiste distinción entre, por un lado la propiedad asociada al capital y por el otro el trabajo como variable de la producción. En este sentido, se considera que el ejercicio de derechos políticos requiere de condiciones materiales que surgen precisamente de una dinámica productiva y de una representación política dirigida por los actores intervinientes en la dinámica productiva que hace al bienestar general. En este sentido, resulta coherente que no haya participación política de los trabajadores en condiciones normales.

85 J. LOCKE, T. T., II, \$27-28; 40-43. Véase en este sentido, J. CHUMBITA, "El desplazamiento en la teoría de la propiedad de John Locke". 